\title{
PENSAMIENTO ESTRATÉGICO: \\ CENTRO NEURÁLGICO DE LA PLANIFICACIÓN ESTRATÉGICA QUE TRANSFORMA LA VISIÓN EN ACCIÓN'
}

\author{
Darcy Luz Mendoza Fernández ${ }^{2}$ - Danny Daniel López Juvinao ${ }^{3}$
}

Universidad de la Guajira, Colombia - Artículo Tipo 2. Reflexión - Recibido: 26 de Mayo 2015 - Aceptado: 24 de Junio 2015

\section{RESUMEN}

El presente artículo de reflexión tiene por objetivo analizar cómo el pensamiento estratégico se constituye en un instrumento valioso para la planificación estratégica organizacional, de tal manera que permita a la empresa alcanzar sus objetivos y resolver problemas dentro de un marco contextual concreto. La metodología utilizada en la investigación es de carácter documental, con apoyo bibliográfico y análisis de la información de autores como Cendrós, Fred, Morrisey, entre otros. Para el pensamiento estratégico el presente no solo es el punto de partida del plan sino su objeto. Es acción con dirección. Se puede concluir que el pensamiento estratégico es el aspecto principal de lo planificado estratégicamente por la organización, conlleva al logro de avances significativos, explora la capacidad de innovación, competitividad y vuelve acción lo que en la planeación es sólo visión.

\section{Palabras Clave:}

Pensamiento estratégico, Planificación estratégica, Acción, Visión.

JEL: O21, L20, L21

\section{Si va a referenciar este artículo}

Mendoza, D. \& López, D. (2015). Pensamiento estratégico: centro neurálgico de la planificación estratégica que transforma la visión en acción. Económicas CUC, 36 (1), 81-94

\footnotetext{
${ }^{1}$ Artículo de reflexión. Derivado del proyecto de investigación: Pensamiento estratégico como base para la responsabilidad social en universidades públicas autónomas. Línea de Investigación: Universidad y Sociedad. Entidad adscrita: Universidad de La Guajira.

${ }^{2}$ Doctora en Ciencias Gerenciales. Magister en Gerencia Empresarial. Licenciada en Administración de Empresas. Docente investigadora de la Universidad de La Guajira. Grupo de investigación: Financo. Email: dmendoza@uniguajira.edu.co

${ }^{3}$ Doctor en Ciencias Gerenciales. Magister en Gerencia Empresarial. Ingeniero en Minas. Docente investigador de la Universidad de La Guajira. Grupo de investigación: Contacto con la comunidad. Email: dlopezj@uniguajira.edu.co
} 


\section{INTRODUCCIÓN}

El presente artículo pretende reflexionar acerca de cómo el pensamiento estratégico hace realidad la planificación estratégica de la organización, tomando como fundamento teórico lo planteado por Cendrós (2001), quien manifiesta que una modalidad del pensamiento estratégico es la que hace énfasis en la planificación, y es aquí donde surge la planificación estratégica que según Fred (2006), abarca el proceso de formulación de estrategias, elaboración de la misión de la empresa, detecta oportunidades y amenazas externas de la organización, definición de fortalezas así como las debilidades, establecimiento de objetivos a largo plazo, generación de estrategias alternativas y la elección de estrategias concretas que se implementarán.

Lo anterior es concomitante con lo que afirma Morrisey (1996), el considera que el pensamiento estratégico se enfoca en los factores más intuitivos del proceso, lo cual, va a permitir la ejecución de la misión, de la visión y de la estrategia de la organización. Esta fase del proceso está diseñada para crear la perspectiva futura de la organización, mientras se establecen los cimientos a partir de los cuales, se tomarán todas las decisiones importantes sobre la planificación. En otras palabras, el pensamiento estratégico se enfoca en los procesos que dan lugar al desarrollo de la misión de la empresa, su visión, sus principios, valores y sus estrategias.

De esta manera, el proceso de la planificación organizacional involucra principalmente tres etapas, la primera el plan operativo, la cual establece planes de acción, de trabajo y presupuestos a un año; la segunda es el plan táctico que hace énfasis en el análisis de asuntos críticos, objetivos y planes de acción también a un año; el tercero es el plan de largo plazo en el que interviene el análisis de asuntos críticos, los objetivos de largo plazo y los planes de acción estratégicos que abarcan de 3 a 5 años, en la cual está inmerso el pensamiento estratégico allí se establecen los valores compartidos, la misión, la visión y la estrategia; esta etapa constituye lo que hoy en día se conoce como la planificación estratégica.

\section{DESARROLLO}

\section{Pensamiento estratégico: \\ Pilar de la estrategia organizacional}

El pensamiento estratégico según Arellano (2004), hace referencia a la forma de pensamiento que está dirigido a la creatividad, imaginación, la búsqueda de alternativas innovadoras; pero con realismo, como potenciación de lo posible enmarcado dentro de un proyecto de construcción intencional de la realidad.

Por su parte, de acuerdo a lo expuesto por Ocaña (2006), el pensamiento estratégico es sinérgico, porque adopta un papel activo que acomoda el afuera al adentro, influyendo sobre el mercado, al tiempo que articula y retroalimenta el marketing con las políticas corporativas; es analítico, flexible, creativo y racional porque establece criterios de valoración para la toma de decisiones, porque se hace menos costoso, se da una máxima rentabilidad o puede mejorar la imagen corporativa, es un sistema de percepción de la realidad que abre nuevas posibilidades competitivas.

Para García (2008), el pensamiento estratégico comporta la creación de una representación del futuro, como esfuerzo científico interdisciplinario, y considera que la prospectiva dará instrumentos para elaborar esas representaciones, pero es, mucho más que una "caja de herramientas". Es, sobre todo, una afirmación de ese futuro que se quiere crear, que se quiere inventar, combinada con una voca- 
ción normativa y una voluntad de allegar los medios necesarios para llevar a cabo las acciones estratégicas que permitirán la realización de ese futuro.

Por consiguiente, tal como lo menciona, el pensamiento estratégico es un sueño que permite visionar hacia donde se quiere llevar a la empresa, este sueño le permite a sus directivas plantear interrogantes como ¿Dónde estaba la empresa ayer?, ¿Dónde está la empresa hoy?, ¿Hacia dónde se quiere llevar a la empresa mañana?, ¿Cómo se hará para conseguirlo?, ¿Cuáles son las fortalezas y debilidades de la empresa?, ¿Cuáles son sus amenazas y oportunidades?; luego entonces, la forma de cómo hacer realidad ese mañana es lo que se denomina la planificación estratégica.

De esta manera, se tiene que el pensamiento estratégico es una forma de visionar a la empresa, utilizando la creatividad, como mecanismo transformador, teniendo claro un horizonte factible, creible y realista, con una prospectiva que lo lleva a evolucionar la actual realidad para lograr con hechos el escenario escogido dentro de las distintas alternativas transformadoras e innovadoras analizadas previamente para dar respuesta a los interrogantes resultantes de investigaciones que dieron origen a ese futuro deseable.

No obstante, Gimbert (2006), indica que para lograr un pensamiento estratégico que apunte a una buena planificación estratégica y cumpla con su finalidad, es necesario llevar a cabo unas fases o etapas, lo cual constituye un proceso en donde deben participar los directivos empresariales, así como los directores de las diferentes áreas funcionales de la organización, lo cual hace de la organización un ente capaz de enfrentarse a los desafíos que el presente siglo trae consigo.

En este mismo sentido, Castañeda (2001), propone la siguiente tabla que identifica las fases del pensamiento estratégico en tres (3) componentes (ver tabla 1), las cuales asocia con una pregunta para luego esbozar los pasos a dar en la planificación estratégica, estas fases 0 etapas condensan las expuestas por el autor mencionado, eso es prever el futuro organizacional, comprenderlo identificando los escenarios estratégicos posibles, para luego después de escoger la mejor opción, implementarlo.

Al considerar esta clasificación, se puede establecer que las teorías y escuelas que abordan el pensamiento estratégico van desde un análisis conceptual hasta un análisis ideológico, con diferentes enfoques y planos de análisis acorde a los cambios que se operan en el entorno, de tal manera que se llegue a la acción después de la visión, de allí que es necesario percibir lo que ocurre en la empresa, comprenderlo para luego entrar a razonar acerca de lo que conviene.

Tabla 1:

Fases del Pensamiento Estratégico

\begin{tabular}{cll}
\hline \multicolumn{1}{c}{ Fase } & Pregunta asociada & \multicolumn{1}{c}{ Pasos de la planificación estratégica } \\
\hline \multirow{2}{*}{ Percepción } & $\begin{array}{l}\text { ¿Que parece estar } \\
\text { ocurriendo aquí? }\end{array}$ & $\begin{array}{l}\text { Visualizar el campo de juego, los participantes y el entorno. } \\
\text { Prever el futuro }\end{array}$ \\
\hline \multirow{2}{*}{ Comprensión } & $\begin{array}{l}\text { ¿Qué posibilidades } \\
\text { enfrentamos? }\end{array}$ & $\begin{array}{l}\text { Identificación de posibilidades estratégicas. } \\
\text { Cotejo de las posibilidades con las capacidades. }\end{array}$ \\
\hline \multirow{2}{*}{ Razonamiento } & ¿Qué haremos al & $\begin{array}{l}\text { Elección de una estrategia principal. } \\
\text { respecto? }\end{array}$ \\
\hline
\end{tabular}

Fuente: Castañeda (2001) 
Sintetizando esta aparte, se entra a precisar que la planificación estratégica implica tanto la intuición como el análisis para establecer las perspectivas futuras que la empresa necesita alcanzar, pero además en su esfuerzo por encontrar una solución al problema estratégico quedaría en gran parte improductivo sin la adecuada inquietud por proporcionar a la empresa las condiciones estructurales e instrumentales necesarias para realizar correctamente la estrategia formulada.

\section{Fundamentos estratégicos del pensamiento: Un norte empresarial para un modelo organizacional}

Los fundamentos estratégicos del pensamiento estratégico, son aquellos elementos que conllevan a la organización a un modelo organizacional coherente con la realidad cambiante de su entorno.

En virtud de ello, Ocaña (2006), considera que estos fundamentos constituyen el norte de la empresa y son el soporte que le permite a la empresa crecer y mantenerse en un mercado flexible. Le da respuesta a interrogantes del presente como ¿Quiénes somos?, ¿Dónde estamos?, también permite interrogarse sobre un futuro queriendo conocer ¿Hacia dónde vamos?, y es con la intervención del plan estratégico que se logra conocer ¿Cómo vamos hacia allá?

En este mismo orden de ideas, Morrisey (1996), señala que el pensamiento estratégico incorpora valores, misión, visión y estrategias elementos intuitivos (basados en los sentimientos) más que analíticos (basados en la información), los cuales se constituyen en fundamentos estratégicos, que al llegar a un acuerdo sobre estos elementos entre los miembros de un equipo administrativo se constituye en un prerrequisito esencial para la planeación efectiva.
Así las cosas, de acuerdo a lo planteado por los autores mencionados, se considera que los elementos fundamentales del pensamiento estratégico que deben existir en las organizaciones se destacan los valores como cimiento organizacional, la visión de futuro como mirada de largo plazo, la misión institucional, las estrategias organizacional como una forma de competencia, y los objetivos estratégicos como atractores para lograr la visión de la organización, estos elementos se analizan a continuación para tener un concepto claro de sus características.

\section{Valores:}

\section{Cimiento organizacional y verdades fundamentales}

Los valores según Dolan \& García (2000), citado por Seijo \& Ávila (2009), constituyen uno de los componentes principales de las formulaciones estratégicas de la organización, son los principios o verdades fundamentales para la organización, a partir de los cuales se estructura su curso de acción y que, según un número creciente de analistas y observadores adquirirán una importancia cada vez mayor en los procesos de planificación y desarrollo organizacional.

En este sentido, Gómez (2009), señala que los valores generalmente tienden a ser elementos que establecen principios de comportamiento de las personas que laboran en una organización, y estos deberían ser cualquier elemento que se considere de vital importancia para lograr cambios importantes y duraderos dentro de la organización, por ejemplo una alianza estratégica en el pensamiento estratégico tradicional, o el trabajo en equipo aunque usualmente se han considerado como valores únicamente el liderazgo, la honradez, la disciplina, etc. 
Serna (1999), sostiene que un proceso de planificación estratégica se inicia por identificar y definir los principios de la organización, los cuales son un conjunto de valores, creencias, normas, que regulan la vida de una organización, definen aspectos importantes de la misma y deben ser compartidos por todos, lo que constituye la norma de vida corporativa y el soporte de la cultura organizacional.

El autor hace una aclaración pertinente acerca de que los principios corporativos no son parte de la visión, ni de la misión, pero son el soporte de las mismas, de allí que cuando se define la visión y misión de la empresa, éstas deben enmarcarse dentro de los principios de la compañía y no pueden ser contrarias. Los principios corporativos, como normas que regulan un comportamiento, son el marco de referencia dentro del cual debe definirse el direccionamiento estratégico de la empresa.

Así las cosas, los valores constituyen para el pensamiento estratégico un elemento esencial porque se consideran el cimiento de la organización, está estrechamente relacionada con la ética, generan beneficios para las personas y para las empresas. En síntesis, los valores definen la personalidad de la organización, hacen parte de la norma de vida corporativa y del soporte de la cultura organizacional como son los principios corporativos conjuntamente con las normas y creencias organizacionales.

\section{Visión:}

\section{Una mirada hacia el futuro}

Piñeiro (2007), considera que el propósito del establecimiento de una visión es expresar los sueños y aspiraciones de las personas que conforman un equipo o una empresa. La declaración de una visión, por definición, está orientada al futuro, esta declaración por lo común incluye palabras como llegar a ser, convertirse, transformarse, esforzarse por. Una buena declaración de visión crea una imagen mental de un estado futuro y esta imagen debe ser compartida por aquellos que la crearon.

Por su parte Soderquist (2005), puntualiza que la visión es ver lo que otros no ven. Una visión es mucho más que una declaración sobre un pedazo de papel puesto en un tablero en el comedor o destacado con un marcador en el informe anual. Es su razón de vivir, debe ser la pasión del líder que debe estar motivado por un fuerte deseo de hacerla realidad, debe impactar todas las decisiones, debe ser algo desafiante paro alcanzable, debe ser emocionante, que su gente se motive para alcanzarla. El papel de un líder es pintar la imagen de esa visión, la tracen y la hagan propia, para que la energía de la organización esté enfocada en el mismo objetivo.

La visión tiene los siguientes elementos, un panorama del futuro que constituye el entorno regulatorio, económico y competitivo para competir; un marco competitivo que lo conforman los negocios y lugares en que la empresa competirá; unos objetivos fundamentales que definen el rol que la empresa adoptará; una descripción de lo que espera lograr; referencias para evaluar el grado de éxito futuro y unas ventajas competitivas que constituyen las habilidades que la empresa desarrollará como apoyo fundamental.

En este marco de ideas, establecer una visión es necesariamente un objetivo a largo plazo, pues conlleva al establecimiento de metas hacia un horizonte lejano que empuja a las personas a lograr más de lo que actualmente están logrando y, necesariamente, el compromiso para hacerla cumplir debe estar impregnado tanto en el líder como en los encargados de llevarla a cabo, es decir, operacionalizarla. 
Establecer una Visión, requiere de unos principios básicos como son proveer una dirección clara y evitar ambigüedades, no focalizarse en la competencia porque limita su alcance, debe ser creativa e innovadora, debe integrar todos los sistemas de la organización. La visión debe ser desafiante pero no imposible.

\section{Misión institucional: \\ La razón de ser de la empresay una manera de inducir comportamientos}

La misión institucional según Seijo \& Ávila (2009), es la enunciación de los propósitos y valores de una organización que la distingue de otras organizaciones en cuanto a sus operaciones, productos, mercados y talento humano que soporta el accionar de la empresa hacia el beneficio, tomando en cuenta al cliente como parte elemental de la actividad de la organización, asimismo, manifiesta que cada organización es única, porque sus principios, valores, misión y filosofía de sus diseños, los colaboradores y los grupos con los que interactúan en el mercado son todos diferentes.

De acuerdo con lo establecido por, la misión es interpretada como la razón de ser de la empresa, una explicación atemporal de la identidad del equipo, el grupo o la organización, la misión define el propósito macro del grupo u organización. Usualmente asume la forma de una declaración simple acerca de lo que la empresa es y cuál es su negocio. En este sentido, el mismo autor considera que la misión no es un objetivo a cumplir, pero es algo más que una explicación ilimitada, es guiar a las personas en el futuro incluso cuando es incierto, provee una razón de ser y justifica su existencia. La declaración de la misión debe ser clara y comprensible, corta, distintiva y alcanzable.
Siguiendo esta lógica, Mintzberg (2007), considera que la Misión es como una afirmación que describe el concepto de la empresa, la naturaleza del negocio, la razón para que exista la empresa, la gente a la que le sirve y los principios y valores bajo los que pretende funcionar. En la Misión se encuentra el fundamento que permite explicar a los demás el sentido de la organización en la sociedad. Una organización sin misión sería como un grupo de amigos que se reúnen porque no tienen nada más que hacer, que no puedan dar cuenta del por qué se han reunido. La Misión es el presente, le da identidad y razón de ser a la organización.

En este mismo sentido, la misión de la empresa, según el mismo autor, se refiere a la forma en que la empresa está constituida, su esencia misma y la relación de ésta con su contexto social, de forma tal que puede definir como una filosofía relacionada con el marco contextual de la sociedad en que opera. Esta a su vez debe ser suficientemente especifica como para servir de guía en el establecimiento de prioridades y en la evaluación del valor estratégico; sin embrago, no debe ser tan específica como para incluir los objetivos y las metas.

Con estas bases conceptuales o fundamentos teóricos descritos, se puede considerar que hablar de la misión institucional es conocer a la empresa y su entorno, tener un amplio espectro de la funcionabilidad institucional, de tal manera que se conjuguen el pasado y el presente en una forma de administración que la empresa logre consolidar con sus colaboradores, de allí que es necesario que la misión responda una serie de preguntas que ayudarán a tener claridad sobre el papel que está llevando a cabo la empresa, que no dé una impresión de contradicción, donde se exprese en papel una misión y la realidad de la institución sea diferente, esto deteriora 
notablemente el clima organizacional y los comportamiento inducidos no sean los más apropiados.

\section{Estrategia Gerencial: Una forma de competencia}

De acuerdo con lo establecido por Garrido (2007), la estrategia es el centro neurálgico de la empresa, es el objeto de conocimiento del pensamiento estratégico, el cual está centrado en torno a la competencia cuyo detonante común se vincula centralmente con la necesidad de triunfar al competir. Así las cosas, el pensar estratégico está puesto en competir para solucionar necesidades en la sociedad para mejorar posición en la participación del mercado.

Para Serna (1999), las estrategias son las acciones que deben realizarse para mantener y soportar el logro de los objetivos de la organización y de cada unidad de trabajo y así hacer realidad los resultados esperados al definir los proyectos estratégicos. Las estrategias son las que permiten concretar y ejecutar los proyectos estratégicos, son el cómo lograr y hacer realidad cada objetivo establecido.

En tal sentido, la estrategia empresarial permite establecer los objetivos de la empresa y los cursos de acción fundamentales de acuerdo a las características del entorno competitivo y de las condiciones concretas actuales y potenciales de la empresa, a fin de lograr que esta última se inserte en el medio socioeconómico al cual pertenece.

Argumenta De León (2000), que es importante reconocer que sin estrategia no existe dirección establecida a seguir, ningún mapa a consultar, ningún plan de acción coherente que produzca resultados deseados y la adaptación de la em- presa al entorno cambiante, de hecho, se convierte en la dirección intencionada del cambio hacia la consecución de la ventaja competitiva en el negocio, donde el medio ambiente impone las reglas de juego, la estrategias potencialmente disponibles para la empresa, encontrándose así, la clave de las distintas habilidades de la organización para enfrentarse a ellas.

Así las cosas, se establece que la estrategia es el nervio central del pensamiento estratégico, pues ella define lo que se piensa desarrollar en el curso de acción, cual es el camino que se piensa seguir y el escenario escogido para accionar lo que ya se tiene visionado, en el medio o entorno a actuar, de manera creativa e innovadora. Es por ello considerada una declaración de intenciones que define dónde desea ubicarse la empresa en el largo plazo, lo cual implica entender los procesos y la manera de evitar dificultades, para luego entrar en competencia, de allí la necesidad de desarrollar un pensamiento estratégico.

\section{Objetivos estratégicos: \\ Atractores para lograr la visión empresarial}

Arellano (2004), considera que los objetivos son una expresión lógica de la relación entre la misión, el diagnóstico para la decisión y el proyecto de integración, como interrelaciones sistemáticas de elementos de la intención y de la constitución de la dirección. Pero, para ser viables y no solo intenciones en su camino racional, los objetivos estratégicos deberán incorporar la lógica de la lucha política y administrativa por la hegemonía de los proyectos alternativos.

Para Iglesias (2004), los objetivos estratégicos se convierten en un conjunto articulado de grandes logros que representan los atractores para lograr la visión a través de un posicionamiento deseado. Para que 
estos objetivos se conviertan en atractores es importante que se satisfagan de manera coherente tres premisas: Que sean valiosos en términos sustantivos, que sean legítimos y políticamente sostenibles, es decir, deben ser capaces de atraer continuamente tanto autoridad como el entorno político autorizador al cual se deben rendir cuentas en último término y que estos puedan traducirse operativamente y sean administrativamente viables.

En este mismo orden de ideas, Serna (1999), enfatiza que los objetivos globales son los resultados a largo plazo que una organización espera lograr para hacer real la misión y la visión de la empresa o área de negocio. Con los objetivos estratégicos se identifican las áreas de énfasis de concentración de todos los esfuerzos de las áreas claves, éstos son el resultado del análisis realizado del entorno, los cuales deben ser mensurables, comprensibles, factibles, conocidos, aceptados, relacionados con la misión y obligatorios para alcanzar la visión.

De esta manera, los objetivos estratégicos conforman una comprensión compartida de aquello que la organización pretende lograr, la manera en que lo logrará, de allí que los objetivos estratégicos de corto y mediano plazo se fijan para cumplir con la misión para trabajar en pos de la visión.

Por lo antes expuesto, se dice que los objetivos estratégicos son las actividades consideradas más importantes para alcanzar el horizonte deseado en el largo plazo, por lo general este plazo es considerado entre dos (2) y cinco (5) años, son los que acompañan a la visión, sirven como guía para el desarrollo de las actividades del día a día, generalmente son más concretos que la visión, deben servir al cumplimiento de la misma, para establecerlos se requiere del pensamiento estratégico de los directivos que conjuntamente con los valores, la misión, la visión y las estrategias constituyen la planificación estratégica.

\section{Planificación estratégica: Un camino largo por recorrer}

Hablar de planificación estratégica en este contexto, es involucrar de manera directa el pensamiento estratégico pues éste es considerado como una herramienta para llevar a cabo todo lo que la organización ha planificado. Así las cosas, se hace necesario explicitar algunas teorías de esta temática expuestas por autores investigadores de la misma.

En este sentido, Fred (2006), acota que la planificación estratégica consiste es decidir sobre en qué negocios se participará, cuáles se abandonarán, cómo se asignarán los recursos, la conveniencia de extender operaciones o diversificarse, el ingreso a mercados internacionales, la fusión o constitución de una empresa de riesgo compartido, entre otros aspectos que le van a permitir a la empresa posicionarse y sostenerse en el mercado.

Burgwal (1999), sostiene que planificar estratégicamente es transformar la realidad, se requiere del análisis de las condiciones del entorno, de los cambios dados en él, las oportunidades que se pueden y se deben aprovechar. Pero, la transformación de la realidad será una empresa infructuosa si al mismo tiempo no se construye una visión de futuro donde señale de manera clara, precisa hacia donde ir y de una misión acompañada cotidianamente de los pasos a dar. En todo caso, los elementos integradores de una estrategia de largo plazo, considerados como una condición para la superación de problemas son: entorno, cambio, oportunidades, visión de futuro y misión. 
De esta manera, la planificación estratégica es el proceso de creación, concepción colectiva de futuro, determinando las maneras de lograrlo, enlazándola con las actividades y responsabilidades individuales para lograr una ejecución óptima, este proceso involucra la visión de la empresa, la misión organizacional, el análisis inter y extra organizacional así como los objetivos estratégicos.

Así las cosas, la empresa u organización podrá establecer a cuales aspectos tendrá que dedicar su mayor esfuerzo, esto necesariamente acarrea unos costos, sin embargo, es el camino más seguro y de menor riesgo porque al final se tendrá la certeza de haber escogido el enfoque con mayor posibilidad de crecimiento empresarial, y los costos se transformarán en beneficios para la empresa.

\section{Proceso de planificación estratégica: Un enfoque global para el logro de las estrategias gerenciales}

La planificación estratégica se realiza a nivel de la organización, es decir, considera un enfoque global de la empresa, por lo que se basa en objetivos y estrategias generales, así como en planes estratégicos, que afectan una gran variedad de actividades, pero que parecen simples y genéricos.

El proceso de planificación estratégica, según Navajo (2009), considera básicamente tres planes a saber: un plan operativo, un plan táctico y un plan estratégico compuesto este por planes de largo plazo y pensamiento estratégico. Las dos primeras fases del proceso se consideran

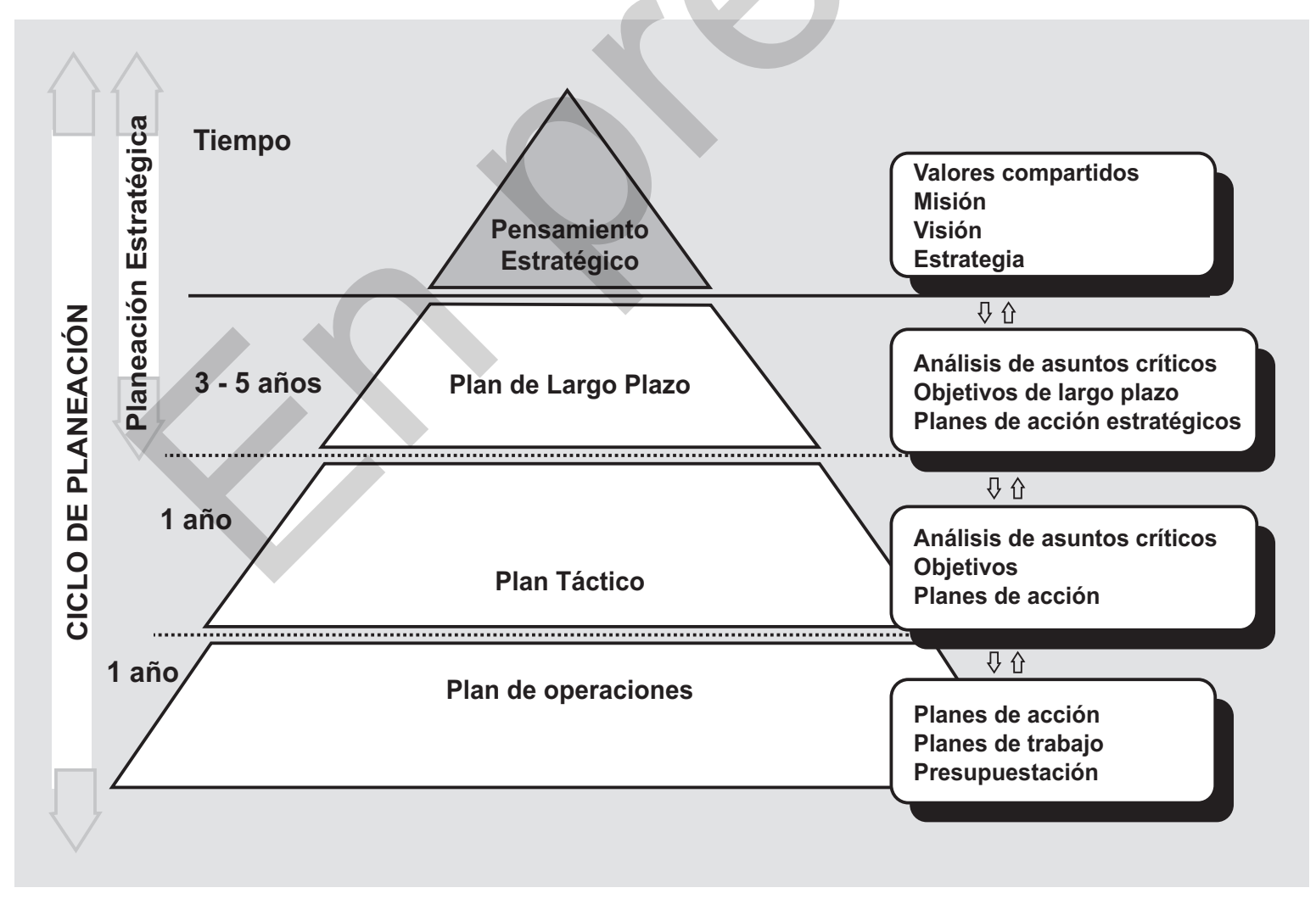

Figura 1: Proceso de Planificación Estratégica 
de corto plazo, pues se desarrollan en un lapso menor de un año y la última fase se desarrolla en un lapso de tiempo mayor a 3 años, es allí donde están inmersos los valores corporativos, la misión, la visión y las estrategias. Tal como se presenta en la figura 1.

En este mismo sentido, Boland (2007), hace mención al proceso de planificación el cual se materializa en diferentes tipos de planes, como son: Planificación estratégica, operativa y táctica. En donde la planeación estratégica es comprensiva, a largo plazo, relativamente general. Los planes estratégicos se centran en temas amplios y duraderos que aseguran la efectividad de la empresa al igual que su supervivencia durante muchos años.

El plan estratégico establece la finalidad de la organización y puede describir un conjunto de metas y objetivos para llevar adelante a la empresa hacia el futuro. Por ejemplo, puede establecer como finalidad la supremacía a nivel mundial en sus áreas específicas de producto, fijando como meta a alcanzar la de introducirse en nuevos mercados basándose en el trabajo de investigación teniendo como objetivo meta al cliente. En este sentido, la planeación estratégica interviene en el análisis de asuntos críticos, objetivos de largo plazo, planes de acción estratégicos, interviene la alta dirección y la organización como un todo, se involucra el pensamiento estratégico, se establecen los valores compartidos, la misión, la visión y la estrategia.

La planificación operativa, se establece a corto plazo, es específica y está orientada a la consecución del objetivo determinado. La planificación operativa convierte los conceptos generales del plan estratégico en cifras claras, en pasos concretos y en objetivos evaluables a corto plazo. La planificación operativa demanda una aplicación de recursos que sea eficiente y efectiva en costes en la solución de problemas y consecución de los objetivos establecidos.

Por su parte, la planificación táctica se ubica en el enlace que puede establecerse entre los procesos de la planificación estratégica y de la planificación operativa. Es más limitada, específica y a medio plazo en comparación con la planificación estratégica. La táctica se refiere más a asuntos relativos a la eficiencia que a la eficacia a largo plazo. Esta planeación hace énfasis en el análisis de asuntos críticos, objetivos y planes de acción a un año, conformada por mandos intermedios, divisiones mayores y sus respectivas funciones.

Así las cosas, el tipo de proceso de planificación que deba seguirse se verán condicionado por el tipo de metas y/o objetivos que se pretenden alcanzar a través del plan. Los objetivos más generales a largo plazo requieren la planificación estratégica; los objetivos precisos de corto plazo son propios de la planificación operativa. Son tres las planificaciones que llevan a un camino de éxito organizacional, las cuales cada una tiene su objetivo, su para qué, se hace necesario que los empresarios enrumben sus organizaciones a través de este camino, y el pensamiento estratégico debe estar presente en la cúspide piramidal puesto que éste da perspectiva, mientras que la planeación de largo plazo conduce a la posición y la planeación táctica conduce al desempeño.

\section{Pensamiento estratégico y planificación: Un sueño hecho realidad}

Como ya se ha esbozado en los acápites anteriores, el pensamiento estratégico es la cúspide del proceso de la planificación estratégica, se presenta entonces a continuación unos ítems donde se refleja la 
relación que existe entre estas dos categorías:

El pensamiento estratégico considera López (2005), proporciona las bases para la planificación estratégica y es en gran medida de naturaleza intuitiva lleva a la perspectiva, mientras que la planificación a largo plazo lleva a la posición y la planificación táctica al rendimiento; el pensamiento estratégico trata los valores, las convicciones filosóficas de los ejecutivos encargados de guiar a su empresa en un viraje exitoso, la misión, visión como conceptos actuales y futuros, la estrategia como la dirección en que debe avanzar la empresa, es el sueño de hacia dónde quiere ir la organización, es la perspectiva, mientras que la planificación estratégica es la forma cómo lograrlo, el plan.

Ocaña (2006), en este mismo sentido analiza otra posición, considera que la planificación estratégica y el pensamiento estratégico son diferentes lados de la misma moneda y cada uno es necesario, pero no suficiente para un marco estratégico eficiente de la gerencia, por eso se hace necesario que el pensamiento estratégico y la planificación deben trabajar de común acuerdo para cosechar la ventaja al máximo.

Queda claro entonces, que una planificación por muy estratégica que sea si no tiene el pensamiento estratégico no se cumplen con esos objetivos que están visionados. El pensamiento estratégico se convierte entonces en esa herramienta para hacer realidad esa planificación en el largo plazo, de hecho, cuando no se tenía claro el uso de esta herramienta, muchas organizaciones no lograban cumplir sus objetivos, de allí que a partir del análisis crítico y concienzudo se inicia en las organizaciones la implementación del pensamiento estratégico en las planeaciones de largo plazo, y es cuando se presentan resultados sorprendentes y el cumplimiento de los objetivos propuestos.

Según análisis realizado sobre la planeación estratégica por Morrisey (1996), la fase de pensamiento estratégico da perspectiva, la planeación a largo plazo conduce a la posición, la planificación táctica conduce al desempeño. El pensamiento estratégico establece qué futuro se espera para la organización. El propósito del pensamiento estratégico es ayudar a explotar los muchos desafíos futuros, tanto previsibles como imprevisibles, más que preparar al directivo para un probable mañana único.

El autor considera que incorporar pensamiento estratégico a la planificación, mantiene concentrada la atención en el futuro y presente; refuerza los principios adoptados tanto en la misión como en visión y estrategia; apoya la comunicación, planificación de funcionamiento holístico, motiva a los directivos a observarla desde un marco perspectivo-prospectivo; ahorra tiempo, reduce el conflicto, aumenta el poder del esfuerzo humano; transforma la planificación a largo plazo en resultados específicos susceptibles de medición; acentúa la planificación del equipo, da a los participantes posesión en el plan y los resultados proyectados, proporciona un medio para llevar a cabo los planes a corto plazo para asegurar la comprensión y compromiso hacia ellos.

Desde esta perspectiva se tiene que en la actualidad muchas organizaciones han realizado su planificación estratégica teniendo en cuenta la incorporación del pensamiento estratégico como un factor que invita a innovar, a pensar en ese futuro de manera holística e integral, siendo incluyente, agregando valor a la sociedad, adoptando términos que la sociedad acoge, incluyendo factores que este mundo globalizado y cambiante exige. 


\section{CONCLUSIONES}

El desarrollo del pensamiento estratégico en una organización conlleva a la creación de un pensamiento dirigido a la creatividad, a la imaginación, a la búsqueda de alternativas innovadoras que permiten visionar el futuro, teniendo en cuenta el pasado sin descuidar el presente, por lo tanto lo sitúa como un nuevo paradigma gerencial que ayuda a conquistar la orientación estratégica haciendo realidad la planificación de este tipo, logrando así una cultura estratégica organizacional.

Por ello, el pensamiento estratégico de una empresa involucra valores, misión, visión al igual que las estrategias constituyendo el direccionamiento estratégico de la organización, que da claridad a la empresa hacia donde se dirige, permite al equipo directivo un análisis prospectivo de los distintos escenarios futuros en el que puede participar para optimizar sus ventajas competitivas en un mercado globalizado.

De allí que la planificación estratégica al ser un proceso en el cual se expone la visión y la misión de la empresa, se involucra necesariamente, el pensamiento estratégico como una herramienta para alcanzar los objetivos organizacionales, sin embargo, el pensamiento estratégico no se debe considerar sólo como una caja de herramientas, puesto que va mas allá debido principalmente a la necesidad de afirmar ese futuro que se quiere crear, que se quiere inventar, para llevar a cabo las acciones estratégicas que permitirán la realización de ese futuro deseable, convirtiendo a la organización en un ente proactivo y anticipatorio con la participación activa de los actores organizacionales.
El pensamiento estratégico se convierte en la cumbre del proceso de la planificación estratégica y vuelve acción lo que en la planeación es sólo visión. El pensamiento estratégico y la planificación son variables intrínsecas, porque sin pensamiento estratégico, la planificación no tiene sentido y sin planificación, el logro de éxito de la gestión sería solo una imaginación, es por eso que la planificación estratégica debe estar sostenida en el pensamiento estratégico y este a su vez en una planificación táctica, que conlleve a hacer realidad el sueño visionado.

La construcción del pensamiento estratégico como parte de un sistema de planificación estratégica debe tomarse con compromiso, esta no debe verse como la elaboración de palabras que concuerdan y se enuncian con cierta locuacidad como la parte cúspide de un discurso, una inspiración o una poesía. El propósito de la administración estratégica es organizar, direccionar, es apoyar las actividades que aunque sean disidentes son a la vez complementarias.

El pensar estratégicamente implica salir de los sistemas convencionales, salir de lo común, es incorporar la lógica del pensamiento, tener la capacidad analítica, ser deductivo, poseer alta dosis de creatividad, ser intuitivo, gran capacidad imaginativa, no ser lineal, ser innovador, descubrir estrategias novedosas. Por su parte, la planificación estratégica es aplicar las estrategias desarrolladas por el pensamiento estratégico, apoyando su proceso.

De esta forma, el pensamiento estratégico supera el problema de la planificación estratégica convencional que ha centrado sus preocupaciones en el futuro, mientras construye el presente por otras vías informales y otros criterios. 
Para el pensamiento estratégico el presente no solo es el punto de partida del plan sino su objeto, su meta, su principal objetivo por el cual se va a trabajar. De allí que el pensamiento estratégico es considerado como un mecanismo que transforma en realidad, en acción, la planificación estratégica, es el centro neurálgico de la misma, que hace tangible lo que se ha planificado estratégicamente, vincula el hoy con el mañana, se formulan programas de acción, la estrategia, es la primera y primordial jerárquicamente dentro del proceso de la planificación estratégica. Es acción con dirección, es hacer hoy lo que visiono ayer y visionar hoy lo que hará mañana. Cuando se sueña de manera planificada se obtienen los resultados esperados, de allí que la recomendación en este sentido es pensar estratégicamente para hacer realidad lo soñado.

\section{REFERENCIAS}

Arellano, D. (2004). Gestión estratégica para el sector público. Recuperado de: http://www.redalyc.org/articulo. oa? id=13314206

Boland, L. (2007), Funciones de la administración. Bogotá: Editorial Mc Graw Hill Interamericana S.A.

Burgwal, G. (1999). Planificación estratégica y operativa, aplicada a gobiernos locales. Quito: Abya Yala Publicaciones.

Castañeda, L. (2001). Pensar: tarea esencial de lideres y gerentes: Aplicación del Pensamiento Multimodal a la toma de decisiones y la solución de problemas organizacionales. Ciudad de México: Panorama Editorial.
Cendrós, J. (2001). Pensamiento Estratégico. Ensayos. Colección Gerencia de la Universidad Rafael Belloso Chacín. Maracaibo: Ediciones Gato Azul.

De León, L. (2000). Pensamiento estratégico, Planeación y Dirección Estratégica. La Habana: Universidad de Cienfuegos.

Dolan, S. \& García, S. (2000). Managing by Values in the Next Milenium: Cultural Redesign for Strategic Organizational Change. Recuperado de: http:// www.econ.upf.es/deehome/what/wpapers/postscripts/486.pdf

Fred, D. (2006). Conceptos de Administración Estratégica. Novena edición. México: Editorial Printice Hall Hispanoamericana, S.A.

García, E. (2008). La Prospectiva en Cuba y su Vinculación con los Esfuerzos de Integración Latinoamericana, $\mathrm{Ob}$ servatorio Cubano de Ciencia y Tecnología. Recuperado de: http://issuu. com/metadata/docs/prospecal/199

Garrido, F. (2007). Pensamiento estratégico. La estrategia como centro neurálgico de la empresa. Barcelona: Ediciones Deusto.

Gimbert, X. (2006). El enfoque estratégico de la empresa: principios y esquemas básicos. Madrid: Edición. Deusto SA.

Gómez, P. (2009). Planificación Estratégica en Organizaciones no Lucrativas. Madrid: Editorial Narcea S.A. Ediciones.

Iglesias, A. (2004). El Planteamiento Estratégico de las Organizaciones Públicas, una visión desde la teoría de caos. Universidad Rey Juan Carlos. Madrid: Editorial Dykinson. 
López, M. (2005). Planeación estratégica de tecnologías informáticas y sistemas de información. Ciudad de México: Editorial Narcea.

Mintzberg, H. (2007). Planeación Estratégica. España: Ediciones Díaz de Santos.

Morrisey, G. (1996). Pensamiento Estratégico. Construya los cimientos de su planeación. Ciudad de México: Editorial Prentice Hall Hispanoamericana.

Navajo, P. (2009). Planificación Estratégica en Organizaciones No Lucrativas. Madrid: Narcea, S.A. de Ediciones.

Ocaña, J. (2006). Pienso luego mi empresa existe. San Vicente: Editorial Club Universitario.
Piñeiro, A. (2007). Pensamiento Estratégico y eficiencia de la Gerencia Pública en las Corporaciones Locales. Negotium, 3(008), 133-156

Seijo, C. \& Ávila M. (2009) Valores éticos y responsabilidad social empresarial: una plataforma gerencial en universidades privadas. Revista Redhecs. 6(4), 26-40.

Serna, H. (1999). Gerencia Estratégica. Planeación y Gestión - Teoría y Metodología (6 Ed.). Colombia: 3R Editores.

Soderquist, D. (2005). El Estilo WalMart. EEUU: Caribe Betania Editores. Editorial Caribe, Inc. U.S.A. 